\title{
Study of identifying code polyhedra for some families of split graphs ${ }^{\star}$
}

\author{
Gabriela Argiroffo ${ }^{1}$, Silvia Bianchi ${ }^{1}$ and Annegret Wagler ${ }^{2}$ \\ \{garua, sbianchi\}@fceia.unr.edu.ar, wagler@isima.fr \\ 1 Facultad de Ciencias Exactas, Ingeniería y Agrimensura \\ Universidad Nacional de Rosario, Rosario, Argentina \\ ${ }^{2}$ University Blaise Pascal (LIMOS, UMR 6158 CNRS), Clermont-Ferrand, France
}

\begin{abstract}
The identifying code problem is a newly emerging search problem, challenging both from a theoretical and a computational point of view, even for special graphs like bipartite graphs and split graphs. Hence, a typical line of attack for this problem is to determine minimum identifying codes of special graphs or to provide bounds for their size. In this work we study the associated polyhedra for some families of split graphs: headless spiders and complete suns. We provide the according linear relaxations, discuss their combinatorial structure, and demonstrate how the associated polyhedra can be entirely described or polyhedral arguments can be applied to find minimum identifying codes for special split graphs. We discuss further lines of research in order to apply similar techniques to obtain strong lower bounds stemming from linear relaxations of the identifying code polyhedron, enhanced by suitable cutting planes to be used in a $\mathrm{B} \& \mathrm{C}$ framework.
\end{abstract}

Keywords: identifying code problem, polyhedral approach, split graphs

\section{Introduction}

Many practical applications can be stated as set covering problems, among them newly emerging search problems for identifying codes [13]. Consider a graph $G=(V, E)$ and denote by $N[i]=\{i\} \cup N(i)$ the closed neighborhood of $i$. A subset $C \subseteq V$ is dominating (resp. identifying) if $N[i] \cap C$ are non-empty (resp. distinct) sets for all $i \in V$. An identifying code of $G$ is a node subset which is dominating and identifying, and the identifying code number $\gamma^{I D}(G)$ of a graph $G$ is the minimum cardinality of an identifying code of $G$.

Determining a minimum identifying code in a graph $G=(V, E)$ can be formulated as set covering problem $\min \mathbf{1}^{T} x, M_{I D}(G) \geq \mathbf{1}, x \in\{0,1\}^{|V|}$ by:

$$
\begin{gathered}
\min \mathbf{1}^{T} x \\
x(N[j])=\sum_{i \in N[j]} x_{i} \geq 1 \quad \forall j \in V \quad \text { (domination) } \\
x(N[j] \triangle N[k])=\sum_{i \in N[j] \triangle N[k]} x_{i} \geq 1 \quad \forall j, k \in V, j \neq k \text { (identification) } \\
x \in\{0,1\}^{|V|}
\end{gathered}
$$

* This work was supported by an ECOS-MINCyT cooperation France-Argentina, A12E01. 
We call

$$
M_{I D}(G)=\left(\begin{array}{c}
N[G] \\
\triangle[G]
\end{array}\right)
$$

the identifying code matrix of $G$, encoding the closed neighborhoods of the nodes of $G(N[G])$ and their symmetric differences $(\triangle[G])$, and define the identifying code polyhedron of $G$ as $P_{I D}(G)=\operatorname{conv}\left\{x \in \mathbb{Z}_{+}^{|V|}: M_{I D}(G) x \geq \mathbf{1}\right\}$. It is clear by construction that $\gamma^{I D}(G)$ equals the covering number $\tau\left(M_{I D}(G)\right):=\min \left\{\mathbf{1}^{T} x\right.$ : $\left.x \in P_{I D}(G)\right\}$. In addition, a graph $G$ has an identifying code or is identifiable if and only if $M_{I D}(G)$ has no zero-row. As $N[G]$ has clearly no zero-row, $G$ is identifiable if and only if $\triangle[G]$ has no zero-row which is equivalent to the known condition that $G$ is identifiable if and only if it has no true twins, i.e., nodes $i, j$ with $N[i]=N[j]$, see [13].

As $M_{I D}(G)$ may contain rows which are equal to or dominated by other rows in $M_{I D}(G)$, we define the corresponding clutter matrix, the identifying code clutter $C_{I D}(G)$ of $G$, obtained by removing repeated or dominated rows from $M_{I D}(G)$. We clearly have that

$$
P_{I D}(G)=\operatorname{conv}\left\{x \in \mathbb{Z}_{+}^{|V|}: C_{I D}(G) x \geq \mathbf{1}\right\},
$$

and obtain as a linear relaxation the fractional identifying code polyhedron

$$
Q_{I D}(G)=\left\{x \in \mathbb{R}_{+}^{|V|}: C_{I D}(G) x \geq \mathbf{1}\right\} .
$$

In $[2,3]$ we characterized when $P_{I D}(G)$ is full-dimensional and which constraints of $Q_{I D}(G)$ define facets of $P_{I D}(G)$ :

Lemma $1([2,3])$. Let $G$ be a graph without isolated nodes and let $V_{1}(G)$ be the set of nodes $k \in V(G)$ such that $\{k\}=N[i] \triangle N[j]$ for two different nodes $i$ and $j$ in $V(G)$. Then,

- $P_{I D}(G)$ is full-dimensional if and only if $V_{1}(G)=\emptyset$.

- The constraint $x_{i} \geq 0$ defines a facet of $P_{I D}(G)$ if and only if $i \notin V_{1}(G)$.

- All constraints from $C_{I D}(G) x \geq \mathbf{1}$ define facets of $P_{I D}(G)$.

Due to the possible formulation as set covering problem, it is immediate that the identifying code problem is hard in general. It even remains hard for several graph classes where many other in general hard problems are easy to solve, including bipartite graphs [6], split graphs [8] and, therefore, chordal graphs (see Section 2 for details).

Our aim is to study identifying codes in split graphs from a polyhedral point of view. In this work we study the associated polyhedra for some families of split graphs: headless spiders and complete suns. We provide the according linear relaxations, discuss their combinatorial structure, and demonstrate how the associated polyhedra can be entirely described or polyhedral arguments can be applied to find minimum identifying codes for special split graphs, see Section 2. We discuss further lines of research in order to apply similar techniques to obtain strong lower bounds stemming from linear relaxations of the identifying code polyhedron, enhanced by suitable cutting planes to be used in a $\mathrm{B} \& \mathrm{C}$ framework, see Section 3. 


\subsection{Preliminary definitions}

Given a set $F$ of vectors in $\{0,1\}^{n}$, we say $y \in F$ is a dominating vector (of $F$ ) if there exits $x \in F$ with $x \leq y$. It can be also said that $x$ is dominated by $y$.

From now on, every matrix has 0,1 -entries, no zero columns and no dominating rows.

As there is a one-to-one correspondence between a vector $x \in\{0,1\}^{n}$ and the subset $S_{x} \subset\{1, \ldots, n\}$ having $x$ as characteristic vector, we write $x$ instead of $S_{x}$. Remind that a cover of a matrix $M$ is a vector $x \in\{0,1\}^{n}$ such that $M x \geq 1$. According to the previous convention, a cover of $M$ is a subset of columns $(\{1, \ldots, n\})$ that intersects all the rows of $M$.

In addition, the cardinality of a cover $x$ is denoted by $|x|$ and equals $\mathbf{1} x$. A cover $x$ is minimum if it has the minimum cardinality and in this case $|x|$ is called the covering number of the matrix $M$, denoted by $\tau(M)$. Recall that the set covering polyhedron of $M$, denoted by $Q^{*}(M)$, is defined as the convex hull of its covers. The polytope $Q(M)=\left\{x \in[0,1]^{n}: M x \geq \mathbf{1}\right\}$ is known as the linear relaxation of $Q^{*}(A)$. When $Q^{*}(A)=Q(A)$ the matrix $A$ is ideal and the set covering problem can be solved in polynomial time (in the size of $M$ ).

A cover of $M$ is minimal if it does not dominate any other cover of $M$. The blocker of $M$, denoted by $b(M)$, is the matrix whose rows are the minimal covers of $M$. It is known that $b(b(M))=M$ and also that a matrix $M$ is ideal if and only if its blocker is (see [14]). In addition, since $b(b(M))=M$ we can refer to $Q^{*}(M)$ and $Q(b(M))$ as a blocking pair of polyhedra. Moreover, $\mathbf{a}$ is an extreme point of $Q(b(M))$ if and only if $\mathbf{a}^{T} x \geq 1$ is a facet defining inequality of $Q^{*}(M)$ (see [11]). In the sequel we will refer to this property as blocking duality.

Given a matrix $M$ and $j \in\{1, \ldots, n\}$, we introduce two matrix operations: the contraction of $j$, denoted by $M / j$, means that column $j$ is removed from $M$ as well as the resulting dominating rows and hence, corresponds to setting $x_{j}=0$ in the constraints $M x \geq \mathbf{1}$. The deletion of $j$, denoted by $M \backslash j$ means that column $j$ is removed from $M$ as well as all the rows with a 1 in column $j$ and this corresponds to setting $x_{j}=1$ in the constraints $M x \geq 1$. Then, given $M$ and $V_{1}, V_{2} \subset\{1, \ldots, n\}$ disjoint, we will say that $M / V_{1} \backslash V_{2}$ is a minor of $M$ and this minor does not depend on the order of operations or elements in $\{1, \ldots, n\}$. It is clear that $M$ is always a minor of itself and we will say that a minor $M / V_{1} \backslash V_{2}$ is proper if $V_{1} \cup V_{2} \neq \emptyset$. It is not hard to see that $b(M / j)=b(M) \backslash j$ and $b(M \backslash j)=b(M) / j$ for every $j \in\{1, \ldots, n\}$. In addition, if a matrix is ideal then so are all its minors (see [7] for further details).

A rank inequality is

$$
\sum_{i \in M^{\prime}} x_{i} \geq \tau\left(M^{\prime}\right)
$$

associated with a minor $M^{\prime}=M \backslash U$. If (1) is a facet of $Q^{*}\left(M^{\prime}\right)$, then it is also a facet of $Q^{*}(M)$ (see [15]).

In addition, if the rank constraint associated with some minor induces a facet defining inequality of $Q^{*}(M)$ then this inequality is also induced by a minor obtained by deletion (see [1] for further details). 


\section{Identifying code polyhedra of some split graphs}

A graph $G=(C \cup S, E)$ is a split graph if its node set can be partitioned into a clique $C$ and a stable set $S$. Hence, split graphs are closed under taking complements by definition. Moreover, they form the complementary core of chordal graphs (graphs without chordless cycles of length $\geq 4$ ) since $G$ is a split graph if and only if $G$ and $\bar{G}$ are chordal [9]. This is also reflected in terms of forbidden subgraphs since a graph is a split graph if and only if it is $\left(C_{4}, \bar{C}_{4}, C_{5}\right)$-free [9] (note that $C_{5}$ is self-complementary and that $\bar{C}_{4}$ occurs as induced subgraph in any chordless cycle $C_{k}$ with $k \geq 6$ such that all chordless cycles $C_{k}$ with $k \geq 4$ are excluded in $G$ as well as in $\bar{G}$ ). The relation between chordal and split graphs can also be interpreted in terms of intersection graphs: while chordal graphs are the intersection graphs of distinct subtrees of a tree, split graphs are the intersection graphs of distinct substars of a star, see e.g. [5].

Our aim is to study identifying codes in split graphs from a polyhedral point of view. First note that a split graph is identifiable if and only if no two nodes in $C$ have the same neighbors in $S$. For instance, a complete split graph (i.e., a split graph where all edges between $C$ and $S$ are present) is not identifiable as soon as $C$ contains 2 nodes (as any two nodes in $C$ are true twins).

Next, recall that finding a minimum identifying code in split graphs is NPhard [8]. So far, $\gamma^{I D}(G)$ is only known for two families: on the one hand, stars (the complete split graphs $G=(C \cup S, E)$ with $|C|=1)$ are the only identifiable complete split graphs and have $\gamma^{I D}(G)=|S|$; on the other hand, split graphs $G=(C \cup S, E)$ where every node in $S$ is connected to a distinct 2-node subset of $C$ have $\gamma^{I D}(G)$ of order $\log (|S|+|C|)$, see [8]. The two families show the wide range of the possible size of minimum identifying codes in split graphs: while the lowest possible lower bound of $\log n$ is attained for the latter, stars achieve almost the highest possible value $n$.

Moreover, a split graph is connected if and only if no node in $S$ is isolated. Every non-connected split graph $G$ contains a connected split graph $G^{\prime}$ and a non-empty subset $S^{\prime} \subset S$ of isolated nodes, and clearly $\gamma^{I D}(G)=\gamma^{I D}\left(G^{\prime}\right)+\left|S^{\prime}\right|$.

This motivates the study of identifying codes in non-complete, connected split graphs $G$. We concentrate on three families of split graphs with a regular structure. This allows us to benefit from a certain combinatorial structure of the identifying code clutter $C_{I D}(G)$ of $G$ and to draw conclusions for the polyhedra $P_{I D}(G)$ and the identifying code number $\gamma^{I D}(G)$ in a similar way as discussed for families of bipartite graphs in [2,3]. In particular, note that stars $K_{1, n}$ are bipartite graphs as well as split graphs. Their identifying code clutter is related to $q$-roses $R_{n}^{q}, 0,1$-matrices with $n$ columns whose rows encode the incidence vectors of all the $q$-element subsets of $\{1, \ldots, n\}$. We have:

Theorem $1([\mathbf{2}, \mathbf{3}])$. For a star $K_{1, n}=(V, E)$ with $n \geq 3$, we have

$-C_{I D}\left(K_{1, n}\right)=R_{n+1}^{2}$;

- $P_{I D}\left(K_{1, n}\right)$ is entirely described by the inequalities $x\left(V^{\prime}\right) \geq|V|-1$ for all nonempty subsets $V^{\prime} \subset V$;

$-\gamma^{I D}\left(K_{1, n}\right)=n$. 
In this paper, we study three families of split graphs with $|S|=|C| \geq 2$ having a regular structure. A headless spider is a split graph $G=(C \cup S, E)$ with $S=\left\{s_{1}, \ldots, s_{n}\right\}, C=\left\{c_{1}, \ldots, c_{n}\right\}$, and $n \geq 2$. In a thin headless spider, $s_{i}$ is adjacent to $c_{j}$ if and only if $i=j$, and in a thick headless spider, $s_{i}$ is adjacent to $c_{j}$ if and only if $i \neq j$. It is straightforward to check that the complement of a thin spider is a thick spider, and vice-versa. Moreover, headless spiders where $s_{i}$ is adjacent to exactly $c_{i}$ and $c_{i+1}$ for all $1 \leq i \leq n$ are called complete suns.

It is easy to see that for $n=2$, the path $P_{4}$ equals the thin and thick headless spider, whereas the complete sun is not identifiable. For $n=3$, the thin headless spider equals the net, and thick headless spider and complete sun its complement, the 3 -sun. We consider headless spiders with $n \geq 4$; Figure 1 illustrates all studied three families for $n=4$. The partition $(C, S)$ is called the spider partition and can be found in linear time [12].

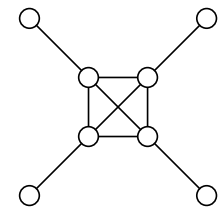

(a)

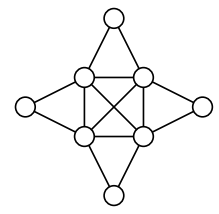

(b)

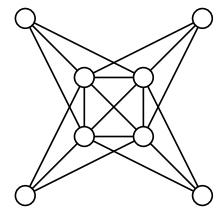

(c)

Fig. 1. (a) thin headless spider, (b) complete sun, (c) thick headless spider.

\subsection{Thick headless spiders}

For simplicity, we will denote thick headless spiders by their partition and we will consider that $C=\{1, \ldots, n\}$ and $S=\{n+1, \ldots, 2 n\}$. Also, E denotes a matrix with all entries at value one.

Lemma 2. For a thick headless spider $G=(C \cup S, E)$ with $n \geq 4$, we have

$$
C_{I D}(G)=\left(\begin{array}{cc}
R_{n}^{n-1} & I \\
0 & R_{n}^{2}
\end{array}\right) .
$$

Proof. Let $G=(C \cup S, E)$ be a thick headless spider. The neighborhood matrix of $G$ can be written as

$$
N[G]=\left(\begin{array}{cc}
\mathbf{E} & R_{n}^{n-1} \\
R_{n}^{n-1} & I
\end{array}\right) .
$$

Now, in order to find $\triangle[G]$ :

(1) If $i, j \in C, N[i] \triangle N[j]=\{i+n, j+n\}$.

(2) If $i, j \in S, N[i] \triangle N[j]=[\{i\} \cup(C-\{i-n\})] \triangle[\{j\} \cup(C-\{j-n\})]=$ $\{i, j, i-n, j-n\}$ and is dominated by a row of the case (1).

(3.a) If $i \in C$ and $j \in S, j \neq i+n, N[i] \triangle N[j]=[C \cup(S-\{i+n\})] \triangle[\{j\} \cup$ $(C-\{j-n\})=\{j-n\} \cup(S-\{j, i+n\})$ and is dominated by a row of the case (1) as $n \geq 4$. 
(3.b) If $i \in C$ and $j=i+n \in S, N[i] \triangle N[j]=[C \cup(S-\{i+n\})] \triangle[\{j\} \cup$ $(C-\{j-n\})=[C \cup(S-\{i+n\})] \triangle[\{i+n\} \cup(C-\{i\})=\{i\} \cup S$ and are dominated by a row of the case (1).

As the first $n$ rows of the matrix $N[G]$ above are also dominated, we have that the clutter matrix $C_{I D}(G)$ can be written as

$$
C_{I D}(G)=\left(\begin{array}{cc}
R_{n}^{n-1} & I \\
0 & R_{n}^{2}
\end{array}\right)
$$

As an immediate consequence, we obtain:

Corollary 1. Let $G=(C \cup S, E)$ be a thick headless spider. Then,

- $P_{I D}(G)$ is full-dimensional.

- The constraint $x_{i} \geq 0$ defines a facet of $P_{I D}(G)$ for each $i \in C \cup S$.

- All constraints from $C_{I D}(G) x \geq \mathbf{1}$ define facets of $P_{I D}(G)$.

Observe that $\tau(I, I)=n$. Then if $G=(C \cup S, E)$ is a thick headless spider, $\gamma^{I D}(G) \geq n$. In fact, we have:

Corollary 2. Let $G=(C \cup S, E)$ be a thick headless spider. Then $S$ is a minimum identifying code and, thus, $\gamma^{I D}(G)=n$.

In [4], the set covering polyhedron $Q^{*}\left(R_{n}^{q}\right)=\operatorname{conv}\left\{x \in \mathbb{Z}_{+}^{n}: R_{n}^{q} x \geq \mathbf{1}\right\}$ of complete $q$-roses was studied.

Theorem 2 ([4]). Let $n \geq q \geq 2$. A non-Boolean inequality ax $\geq 1$ is a facet defining inequality for $Q^{*}\left(R_{n}^{q}\right)$ if and only if ax $\geq 1$ can be written as $x\left(A_{s}\right) \geq$ $\left|A_{s}\right|-q+1$ for some $A_{s} \subset\{1, \ldots, n\}$ where $s \in\{0, \ldots, n-q-1\}$ and $\left|A_{s}\right|=n-s$.

As $R_{n}^{2}$ is a minor of $C_{I D}(G)$ obtained from the deletion of the nodes of $C$, in the underlying graph $G=(C \cup S, E)$, we have:

Corollary 3. Let $G=(C \cup S, E)$ be a thick headless spider. Then, for all nonempty subsets $A \subset S$, the inequalities $x(A) \geq|A|-1$ are facets of $P_{I D}(G)$.

In order to study the remaining facets we need a description of the blocker of $C_{I D}(G)$.

From now on we consider vectors in $\{0,1\}^{l+k}$ of the form $e_{i} \oplus f_{j}$ where $e_{i}$ for $i=1, \ldots, l$ is the unit vector in $\{0,1\}^{l}$ and $f_{j}$ for $j=1, \ldots, k$ is a vector in $\{0,1\}^{k}$ such that $\left(f_{j}\right)_{t}=0$ if $j=t$ and $\left(f_{j}\right)_{t}=1$ if $j \neq t$.

Theorem 3. Let $C_{I D}(G)$ be the clutter matrix of a thick headless spider $G=$ $(C \cup S, E)$. Every minimal cover $x$ of $\left.C_{I D}(G)\right)$ is minimum. Moreover, either $x=\mathbf{0} \oplus \mathbf{1}$ where $\mathbf{0}, \mathbf{1} \in\{0,1\}^{n}$ or $x=e_{i} \oplus f_{j}$ where $e_{i}, f_{j} \in\{0,1\}^{n}$ with $i \neq j$.

Proof. From Corollary 2, $S$ is a minimum cover of $C_{I D}(G)$ ).

Now, let $x$ be a minimal cover of $\left.C_{I D}(G)\right)$ such that $x_{j}=0$ for some $j \in$ $\{n+1, \ldots, 2 n\}$. It is known that every row of $R_{n}^{n-1}$ covers $\left(e_{i}+e_{j}\right)$ for every $i, j=1, \ldots, n[4]$, then any cover $x$ with $x_{n+j}=0$ for some $j \in\{n+1, \ldots, 2 n\}$ 
must be of the form $x=y \oplus f_{j}$ with some $y \in\{0,1\}^{n}$. In order to cover the rows of submatrix $\left(R_{n}^{n-1}, I\right)$ it is enough to consider $x^{i, j}=e_{i} \oplus f_{j}$ for $i, j=1, \ldots, n$ and $i \neq j$. Then $|x|=\left|x^{i, j}\right|=n$ for every $i, j=1, \ldots, n$ and $i \neq j$ and they are all minimum covers.

Now, let $v$ be a cover of $\left.C_{I D}(G)\right)$, with $v_{n+j}=0$. Then $v=y \oplus f_{j}$ and $y \in\{0,1\}^{n}$. But $y$ must be a cover of $R_{n}^{n-1}$, i.e., $y=e_{i}+h$ for some $i \neq j$ and some $h \in\{0,1\}^{n}$. Then $y$ is not minimal.

We can further prove the following:

Corollary 4. If $\bar{x} \in \mathbb{R}^{2 n}$ is an extreme point of $Q\left(b\left(C_{I D}(G)\right)\right)$ such that $x_{i} \neq 0$ then $\bar{x}=\frac{1}{n} \mathbf{1} \in \mathbb{R}^{2 n}$.

Proof. From Theorem 3 it follows that every row of $b\left(C_{I D}(G)\right)$ is either $\mathbf{0} \oplus \mathbf{1}$ where $\mathbf{0}, \mathbf{1} \in\{0,1\}^{n}$ or $e_{i} \oplus f_{j}$ for $i, j=1, \ldots, n$ and $i \neq j$. Then they all have $n$ ones per row. One can show that there are $2 n$ linearly independent rows. It follows that if $\bar{x}=\frac{1}{n} \mathbf{1} \in \mathbb{R}^{n}$ then it satisfies $b\left(C_{I D}(G)\right) \bar{x}=\mathbf{1}$. Hence $\bar{x}$ is a fractional extreme point of $Q\left(b\left(C_{I D}(G)\right)\right)$. Now, if $\bar{y}$ is an extreme point of $Q\left(b\left(C_{I D}(G)\right)\right)$ with all nonzero components then it must satisfy $2 n$ linearly independent inequalities of $Q\left(b\left(C_{I D}(G)\right)\right)$ at equality. It follows that $\bar{y}=\bar{x}$.

Using blocking duality it can be seen that Corollary 4 gives an alternative proof of $\gamma^{I D}(G)=n$ and states that the only facet of $P_{I D}(G)$ with full support is the rank inequality associated with $C_{I D}(G)$.

With the help of some technical lemmas, we can further show:

Theorem 4. Let $b\left(C_{I D}(G)\right)$ be the blocker of the identifying clutter matrix of a thick headless spider $G=(C \cup S, E)$. Let $\bar{x} \in \mathbb{R}^{2 n}$ be a fractional extreme point of $Q\left(b\left(C_{I D}(G)\right)\right)$ such that the set $A=\left\{i: \bar{x}_{i}=0\right\}$ is nonempty. Then either

1. $A \subsetneq S$ and $\bar{x}_{i}=\frac{1}{n-|A|}$ when $i \notin A$ or

2. $C \subsetneq A$ and $|A| \leq 2(n-1)$ and $\bar{x}_{i}=\frac{1}{|A-C|-1}$ for all $i \notin A$.

As a consequence of Theorem 4 and blocking duality, we conclude:

Corollary 5. Let $G=(C \cup S, E)$ be a thick headless spider and $S^{\prime} \subset S$ nonempty. Then, the inequalities $x(C)+x\left(S^{\prime}\right) \geq n-\left|S-S^{\prime}\right|$ when $2 \leq\left|S^{\prime}\right| \leq n-1$ and $x\left(S^{\prime}\right) \geq\left|S^{\prime}\right|-1$ when $2 \leq\left|S^{\prime}\right| \leq n$ are facets of $P_{I D}(G)$.

As a consequence of Corollary 5 and Theorem 4, we obtain the main result of this section:

Corollary 6. Let $G=(C \cup S, E)$ be a thick headless spider. Then, the facets of $P_{I D}(G)$ are:

- the constraint $x_{i} \geq 0$ for all $i \in C \cup S$;

- the constraints $C_{I D}(G) x \geq \mathbf{1}$;

- the constraints $x(C)+x\left(S^{\prime}\right) \geq n-\left|S-S^{\prime}\right|$ and $x\left(S^{\prime}\right) \geq\left|S^{\prime}\right|-1$ for every $S^{\prime} \subseteq S$ with $2 \leq\left|S^{\prime}\right|$. 


\section{$2.2 \quad$ Thin headless spiders}

Lemma 3. For a thin headless spider $G=(C \cup S, E)$ with $n \geq 4$, we have

$$
C_{I D}(G)=\left(\begin{array}{cc}
I & I \\
0 & R_{n}^{2} \\
R_{n}^{n-1} & 0
\end{array}\right) .
$$

Proof. Let $G=(C \cup S, E)$ be a thin headless spider having $C=\{1, \ldots, n\}$ and $S=\{n+1, \ldots, 2 n\}$. The neighborhood matrix of $(C, S)$ can be written as

$$
N[G]=\left(\begin{array}{cc}
\mathbf{E} & I \\
I & I
\end{array}\right) .
$$

Now, in order to find $\triangle[G]$ :

(1) If $i, j \in C, N[i] \triangle N[j]=\{i+n, j+n\}$. $N[i]$.

(2) If $i, j \in S, N[i] \triangle N[j]=\{i, i-n\} \triangle\{j, j-n\}$ and are dominated by

(3.a) If $i \in C$ and $j \in S, j \neq i+n, N[i] \triangle N[j]=[C \cup\{i+n\})] \triangle\{j, j-n\}=$ $\{j, i+n\} \cup(C-\{j-n\})$ is dominated by $\mathrm{N}[\mathrm{i}]$.

(3.b) If $i \in C$ and $j=i+n \in S, N[i] \triangle N[j]=[C \cup\{i+n\})] \triangle[\{i, i+n\}=$ $C-\{i\}$.

As the first $n$ rows of the matrix $N$ above are also dominated, we have that the clutter matrix $C_{I D}(G)$ can be written as

$$
C_{I D}(G)=\left(\begin{array}{cc}
I & I \\
0 & R_{n}^{2} \\
R_{n}^{n-1} & 0
\end{array}\right) .
$$

As an immediate consequence, we obtain:

Corollary 7. Let $G=(C \cup S, E)$ be a thin headless spider. Then,

- $P_{I D}(G)$ is full-dimensional.

- The constraint $x_{i} \geq 0$ defines a facet of $P_{I D}(G)$ for all $i \in C \cup S$.

- All constraints from $C_{I D}(G) x \geq \mathbf{1}$ define facets of $P_{I D}(G)$.

Observe that $\tau(I, I)=n$. Then if $G=(C \cup S, E)$ is a thin headless spider, $\gamma^{I D}(G) \geq n$. In fact, we have:

Corollary 8. Let $G=(C \cup S, E)$ be a thin headless spider. Then, $\gamma^{I D}(G)=$ $n+1$.

Moreover, we obtain:

Corollary 9. Let $G=(C \cup S, E)$ be a thin headless spider. Then,

1. the inequalities $x(A) \geq|A|-1$ for all nonempty subsets $A \subset S$ are facets of $P_{I D}(G)$,

2. the inequality $x(C) \geq 2$ is a facet of $P_{I D}(G)$. 
Proof. As $R_{n}^{2}$ is a minor of $C_{I D}(G)$ obtained after deletion of the nodes in $C$, as a consequence of Theorem 2 we have that the inequalities $x(A) \geq|A|-1$ for all nonempty subsets $A \subset S$ are facets of $P_{I D}(G)$.

Also, $R_{n}^{n-1}$ is a minor of $C_{I D}(G)$ obtained after deletion of the nodes in $S$, and again using Theorem 2 we obtain $x(C) \geq 2$ as a facet of $P_{I D}(G)$.

As an immediate observation the rank inequality $x(C, S) \geq n+1$ is not a facet of $P_{I D}(G)$ since it can be obtained as the sum of the facets $x(S) \geq n-1$ and $x(C) \geq 2$.

Based on our computational experience, we conjecture that the identifying code polyhedra $P_{I D}(G)$ of thin headless spiders have rank facets of a special structure only:

Conjecture 1. Let $G=(C, S)$ be a thin headless spider. Then, the facets of $P_{I D}(G)$ are:

- the constraint $x_{i} \geq 0$ for all $i \in C \cup S$;

- all constraints from $C_{I D}(G) x \geq \mathbf{1}$;

- the constraint $x(C) \geq 2$;

- the constraints $x\left(S^{\prime}\right) \geq\left|S^{\prime}\right|-1$ for all nonempty subsets $S^{\prime} \subset S$.

\subsection{Complete suns}

As third family of headless spiders $G=(C \cup S, E)$ having a regular structure, we consider complete suns, where $S=\left\{s_{1}, \ldots, s_{n}\right\}, C=\left\{c_{1}, \ldots, c_{n}\right\}$ and $s_{i}$ is adjacent to exactly $c_{i}$ and $c_{i+1}$ for all $1 \leq i \leq n$ (indices are taken modulo $n$ ).

In contrary to thin and thick headless spiders whose identifying code clutters are composed by few $q$-roses, the identifying code clutters of complete suns have a more complex structure, involving different combinations of submatrices with a circular structure, where some submatrices occur for all $n \geq 4$, others not (depending on the parity of $n$ and the size of the graph).

A circulant matrix is a square matrix where each row vector is rotated one element to the right relative to the preceding row vector. We denote by $C_{n}^{k}$ a matrix in $\{0,1\}^{n \times n}$ having as first row the vector starting with $k 1$-entries and having 0 -entries otherwise. Moreover, we denote by $C_{n}^{k+k}$ a matrix in $\{0,1\}^{n \times n}$ with $n \geq 2 k+2$ having as first row the vector starting with $k$ 1-entries, then having 0 -entries, again $k 1$-entries, and 0 -entries otherwise.

Lemma 4. For a complete sun $G=(C \cup S, E)$ with $n \geq 4$, the identifying code clutter $C_{I D}(G)$ is composed by the following submatrices

\begin{tabular}{|c|c|c|}
\hline$C_{n}^{2}$ & $I$ & $\forall n \geq 4$ \\
\hline 0 & $\left.C_{n}^{1+1}\right)$ & $\forall n \geq 5$ \\
\hline 0 & $\left.\mid C_{\frac{n}{2}}^{1}, C_{\frac{n}{2}}^{1}\right)$ & for $n=4$ \\
\hline 0 & $\left(C_{n}^{2+2^{2}}\right)$ & $\forall n \geq 9$ \\
\hline 0 & $\left.\mid C_{\frac{n}{2}}^{2}, C_{\frac{n}{2}}^{2}\right)$ & $\forall n \geq 8, n$ even \\
\hline$C_{n}^{n-2}$ & $I \quad)$ & $\forall n \geq 4$ \\
\hline$C_{n}^{1+1}$ & | $\left.\quad C_{n}^{2} \quad\right)$ & $\forall n \geq 4$ \\
\hline
\end{tabular}


where the first part refers to $C$, the second part of the matrices to $S$.

Proof. Let $G=(C \cup S, E)$ be a complete sun. The neighborhood matrix $N[G]$ of $(C, S)$ is composed from

$$
\begin{array}{ll}
\left(\mathbf{E} \mid C_{n}^{2}\right) & \text { for } N[C], \\
\left(C_{n}^{2} \mid I\right) & \text { for } N[S] .
\end{array}
$$

Thus, only $N[S]$ is in $C_{I D}(G)$. In order to find $\triangle[G]$, we distinguish three cases. Case 1: the symmetric differences between two nodes in $C$ have the form

$-N\left[c_{i}\right] \triangle N\left[c_{i+1}\right]=\left\{s_{i-1}, s_{i+1}\right\}$

$-N\left[c_{i}\right] \triangle N\left[c_{i+j}\right]=\left\{s_{i-1}, s_{i}, s_{i+j-1}, s_{i+j}\right\}$ for $1<j \leq \frac{n}{2}$.

For all $n \geq 4$, the former symmetric differences remain in $C_{I D}(G)$ as submatrix

$$
\left(0 \mid C_{n}^{1+1}\right) \quad \forall n \geq 5
$$

(but yield for $n=4$ not the whole circulant matrix). The latter symmetric differences are dominated by the former if $j=2,3$. Thus, for each $4 \leq j \leq \frac{n}{2}$, the symmetric differences $N\left[c_{i}\right] \triangle N\left[c_{i+j}\right]$ remain in $C_{I D}(G)$ as submatrix

$$
\left(0 \mid C_{n}^{2+2}\right) \quad \forall n \geq 9
$$

(but yield for $j=\frac{n}{2}$ not the whole circulant matrix).

Case 2: the symmetric differences between nodes in $C$ and $S$ have the form

- N $\left[c_{i}\right] \triangle N\left[s_{i}\right]=\left(C-\left\{c_{i}, c_{i+1}\right\}\right) \cup\left\{s_{i-1}\right\} ;$

$-N\left[c_{i}\right] \triangle N\left[s_{i-1}\right]=\left(C-\left\{c_{i-2}, c_{i-1}\right\}\right) \cup\left\{s_{i}\right\}$;

- N[cic $\left.\triangle c_{i}\right] N\left[s_{j}\right]=\left(C-\left\{c_{j-1}, c_{j}\right\}\right) \cup\left\{s_{i-1}, s_{i}\right\}$ for $j \neq i, i-1$.

Thus, $N\left[c_{i}\right] \triangle N\left[s_{j}\right]$ is dominated by $N\left[s_{i}\right]$ if $j \neq i, i-1$, and remains in $C_{I D}(G)$ for $j=i, i-1$, forming two submatrices of the form

$$
\left(C_{n}^{n-2} \mid I\right) \quad \forall n \geq 4 .
$$

Case 3: the symmetric differences between two nodes in $S$ have the form

- N $\left[s_{i}\right] \triangle N\left[s_{i+1}\right]=\left\{c_{i}, c_{i+2},\right\} \cup\left\{s_{i}, s_{i+1}\right\} ;$

$-N\left[s_{i}\right] \triangle N\left[s_{j}\right]=\left\{c_{i}, c_{i+1}, c_{j}, c_{j+1}\right\} \cup\left\{s_{i}, s_{j}\right\}$ for $j \neq i$.

Thus, $N\left[s_{i}\right] \triangle N\left[s_{j}\right]$ is dominated by $N\left[s_{i}\right]$ if $j \neq i+1$, and remains in $C_{I D}(G)$ for $j=i+1$, forming a submatrix of the form

$$
\left(C_{n}^{1+1} \mid C_{n}^{2}\right) \quad \forall n \geq 4 .
$$

This together completely describes the identifying code clutter $C_{I D}(G)$.

As an immediate consequence, we obtain:

Corollary 10. Let $G=(C \cup S, E)$ be a complete sun with $n \geq 4$. 
- $P_{I D}(G)$ is full-dimensional.

- The constraint $x_{v} \geq 0$ defines a facet of $P_{I D}(G)$ for each $v \in C \cup S$.

- All constraints from $C_{I D}(G) x \geq \mathbf{1}$ define facets of $P_{I D}(G)$.

Unfortunately, the whole system of facet-defining inequalities for the identifying code polyhedra $P_{I D}(G)$ of complete suns is not easy to describe since non-rank facets are required for all cases $n \geq 4$ (in fact, most facets of $P_{I D}(G)$ are non-rank and involve large coefficients). However, from a careful analysis of the constraints involved in the identifying code clutter $C_{I D}(G)$ of complete suns, we derive at the following conjecture:

Conjecture 2. For a complete sun $G=(C \cup S, E)$ with $n \geq 4$, the stable set $S$ is a minimum identifying code.

Note that it is easy to see that $S$ is always an identifying code for a complete sun $G=(C \cup S, E)$, since all rows of $C_{I D}(G)$ have at least one 1-entry in $S$. Hence, $\gamma^{I D}(G) \leq|S|=n$ follows. On the other hand, for some cases, it has been already verified that $S$ is a minimum identifying code, by generating the full rank constraint $x(C)+x(S) \geq|S|=n$ by means of the Chátal-Gomory procedure. This implies $\gamma^{I D}(G) \geq|S|=n$, and together equality follows for these cases. Our goal is to find a general construction of this type for all $n \geq 4$.

\section{Concluding remarks}

The identifying code problem is hard in general and challenging both from a theoretical and a computational point of view, even for special graphs like split graphs [8]. In this paper, we studied three families of split graphs with $|S|=|C| \geq 2$ having a regular structure: thin headless spiders, thick headless spiders, and complete suns. For all three families, we determined the identifying code clutter and discussed according consequences. In the case of thin and thick spiders $G$, $C_{I D}(G)$ is composed from certain $q$-roses. Based on related results from $[4,15]$, we could give the complete description of $P_{I D}(G)$ for thick spiders, and arrived at a profound conjecture for thin spiders. For both classes, we found the exact value for $\gamma^{I D}(G)$ : $|S|$ for thick spiders and $|S|+1$ for thin spiders. It turned out that the identifying code clutters of complete suns have a more complex structure involving different circulant matrices and, accordingly, more involved facets are required to describe $P_{I D}(G)$. For this class, we showed $\gamma^{I D}(G) \leq|S|$ and conjecture that $\gamma^{I D}(G)=|S|$ holds. So, all three families seem to have small minimum identifying codes close to the lower bound of order $\log (|S|+|C|)$.

This demonstrates how the polyhedral approach can be applied to find identifying codes of minimum size for special graphs $G$, just by determining and analyzing the identifying code clutter $C_{I D}(G)$, even in cases where no complete description of $P_{I D}(G)$ is known yet.

As future lines of research, we plan to apply similar and more advanced techniques to obtain either the identifying code of minimum size or strong lower bounds stemming from linear relaxations of the identifying code polyhedron, 
enhanced by suitable cutting planes. For that, note that facets associated with deletion minors of $C_{I D}(G)$ remain facets in $P_{I D}(G)$, so according facets identified for special graphs are relevant for every graph having such subgraphs.

\section{References}

1. Argiroffo, G., Bianchi, S.: On the set covering polyhedron of circulant matrices, Discrete Optimization 6(2), 162-173 (2009)

2. Argiroffo, G., Bianchi, S., Wagler, A.: Polyhedra associated to identifying codes (extended abstract), In: Proc. of the VII Latin-American Algorithms, Graphs and Optimization Symposium (LAGOS 2013), Electronic Notes in Discrete Mathematics 44, 175-180 (2013)

3. Argiroffo, G., Bianchi, S., Wagler, A.: Polyhedra associated with identifying codes, submitted to Discrete Applied Mathematics

4. Argiroffo, G., Carr, M.: On the set covering polyhedron of $q$-roses, In: Proc. of the VI ALIO/EURO Workshop on Applied Combinatorial Optimization 2008, Buenos Aires, Argentina (2008).

5. Brandstdt, A., Le, V.B., Spinrad, J.: Graph Classes: A Survey, SIAM Monographs on Discrete Mathematics and Applications (1999)

6. Charon, I., Hudry, O., Lobstein, A.: Minimizing the size of an identifying or locating-dominating code in a graph is NP-hard, Theoretical Computer Science 290, 2109-2120 (2003)

7. Cornuéjols, G.: Combinatorial Optimization: Packing and Covering, SIAM, CBMS $74(2001)$

8. Faucoud, F.: The complexity of the identifying code problem in restricted graph classes, In: Proc. of the International Workshop on Combinatorial Algorithms (IWOCA 2013), LNCS, vol. 8288, pp. 150-163. Springer, Heidelberg (2013)

9. Földes, S., Hammer, P.: Split graphs, In: Proc. of the VIII Southeastern Conference on Combinatorics, Graph Theory and Computing (Baton Rouge, La.), Congressus Numerantium XIX, Winnipeg: Utilitas Math., pp. 311-315, (1977)

10. F. Foucaud, G. Mertzios, R. Naserasr, A. Parreau and P. Valicov. Identifying codes in subclasses of perfect graphs. In preparation.

11. Fulkerson, D.: Blocking polyhedra, In: B. Haris (Ed.), Graph Theory and its Applications, pp. 93-112. Academic Press, New York (1970)

12. Jamison, B., Olariu, S.: Recognizing $P_{4}$-tidy graphs in linear time, SIAM Journal on Comput. 21, 381-406 (1992)

13. Karpovsky, M.G., Chakrabarty, K., Levitin, L.B.: On a new class of codes for identifying vertices in graphs, IEEE Transactions on Information Theory 44, 599611 (1998)

14. Lehman, A.: On the width-length inequality, Mathematical Programming 17, 403417 (1979)

15. Sassano, A.: On the facial structure of the set covering polytope, Mathematical Programming 44, 181-202 (1989) 